\title{
Synthesis and Antidiabetic Evaluation of Benzothiazole Derivatives
}

\author{
Mariappan G*, Prabhat P, Sutharson L, Banerjee J, Patangia U, and Nath S \\ Department of Medicinal Chemistry, Himalayan Pharmacy Institute, Majhitar, Rangpo, \\ East Sikkim-737136, India. "E-mail: gmariappanhpi@yahoo.co.in
}

(Received February 18, 2012; Accepted March 27, 2012)

\begin{abstract}
A novel series of benzothiazole derivatives were synthesized and assayed in vivo to investigate their hypoglycemic activity by streptozotocin-induced diebetic model in rat. These derivatives showed considerable biological efficacy when compared to glibenclamide, a potent and well known antidiabetic agent as a reference drug. All the compounds were effective, amongst them 3d showed more prominent activity at $100 \mathrm{mg} / \mathrm{kg}$ p.o. The experimental results are statistically significant at $\mathrm{p}<0.01$ and $\mathrm{p}<0.05$ level.
\end{abstract}

Key words: Benzothiazole, Hypoglycemic activity, Riluzole, Antitumor activity, Diabetes

\section{INTRODUCTION}

Benzothiazole ring system is present in various marine and terrestrial natural compounds, which have useful biological activities. ${ }^{1-4}$ 2-Aminobenzothiazoles are highly reactive compounds and extensively utilized as reactants or reaction intermediates since the $\mathrm{NH}_{2}$ and endocyclic $\mathrm{N}$ functions are suitably situated to enable reactions with various reactants to form a variety of fused heterocyclic compounds. Medicinal chemist attention was drawn to this series when the pharmacological profile of Riluzole ${ }^{5}$ was observed as clinically available anticonvulsant drug. These derivatives are reported in the literature for the treatment of epilepsy, ${ }^{6-12}$ inflammation, ${ }^{13,14}$ analgesia, ${ }^{15,16}$ amyotrophic lateral sclerosis, ${ }^{17}$ and viral infections. ${ }^{18}$ They also exhibit antitumour, ${ }^{19-33}$ antitubercular, ${ }^{34,35}$ antibacterial, ${ }^{36}$ antifungal,${ }^{37}$ antimalarial, ${ }^{38}$ antihelmintic. ${ }^{39}$ 2-aryl substituted benzothiazoles have emerged in recent years as an important pharmacophore in non-invasive diagnosis of alzheimer's disease. ${ }^{40}$ Benzothiazole derivatives have been evaluated as potential amyloid-binding diagnostic agents in neurodegenerative disease ${ }^{41,42}$ and selective fatty acid amide hydrolase inhibitors. ${ }^{43}$ They are broadly found in bio organic and medicinal chemistry with applications in drug discovery and development for the treatment of diabetes. ${ }^{44-52}$ Diabetes has become an increasing concern to the world's population. In view of these literatures, it was of considerable interest to synthesize the title compound with a hope to obtain potent biologically active and safe oral anti diabetic agents.

\section{RESULT AND DISCUSSION}

\section{Chemistry}

A congeneric series of benzothiazole derivatives were synthesized as illustrated in Scheme 1. The starting mate-

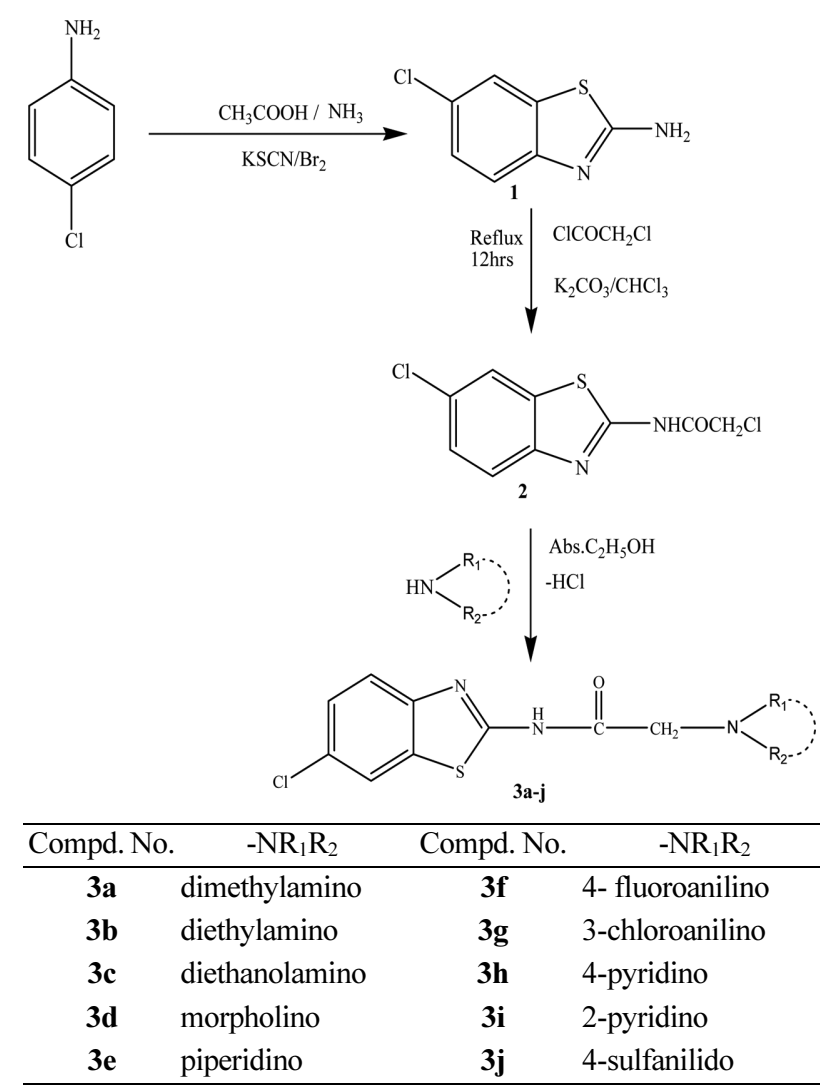

Scheme 1. Synthesis of benzothiazole derivatives. 
Table 1. Physical data of the synthesized compounds 3(a-j)

\begin{tabular}{ccc}
\hline Comp. No & Yield (\%) & M.P $\left({ }^{\circ} \mathrm{C}\right)$ \\
\hline 3a & 80 & $196-199$ \\
3b & 73 & $190-192$ \\
3c & 85 & $201-202$ \\
3d & 83 & $156-158$ \\
3e & 77 & $193-195$ \\
3f & 67 & $198-200$ \\
3g & 61 & $193-195$ \\
3h & 68 & $190-193$ \\
3i & 61 & $167-169$ \\
3j & 69 & $204-206$ \\
\hline
\end{tabular}

rial 2-amino-5-chloro benzothiazole 1 was prepared by the reaction of 4-chloro aniline and potassium thiocyanate in glacial acetic acid. The compound $\mathbf{1}$ was refluxed with chloroacetyl chloride in presence of potassium carbonate and chloroform to yield 2-chloroacetamido-5chloro-benzothiazole $\mathbf{2}$. The condensation of compound $\mathbf{2}$ with various primary and secondary amines in absolute alcohol and $\mathrm{HCl}$ afforded the final compounds $\mathbf{3}(\mathbf{a}-\mathbf{j})$. Their structures have been elucidated from UV, IR, ${ }^{1} \mathrm{H}$ NMR, mass spectral data and elemental analysis. The physicochemical data of the synthesized compounds are given in Table 1.

\section{Spectral Analysis}

A sharp singlet at $2.52-3.40$ ppm ascertained the presence of $\mathrm{CH}_{2}$ proton (aliphatic) and a characteristic signal at 7.14-7.60 ppm is assigned to NH proton (benzothiazole) in all the synthesized compounds. A multiplet observed at $6.52-8.15 \mathrm{ppm}$ indicated the presence of aromatic proton in all the compounds. In addition to this, two $\mathrm{OH}$ proton of compound $3 \mathbf{c}$ exhibited a sharp singlet at $2.48 \mathrm{ppm}$. The two sharp singlet signals of morpholine were observed at 2.52 and 3.55 ppm corresponding to two types of $\mathrm{CH}_{2}$ proton in compound 3e. The appearance of signals at 1.53 ppm and $2.50 \mathrm{ppm}$ ascertained the presence of $\mathrm{CH}_{2}$ of piperidine. A sharp singlet at $4.06 \mathrm{ppm}, 4.46 \mathrm{ppm}$ and 4.49 ppm demonstrated the existence of $\mathrm{NH}$ proton (benzene) for the compounds $\mathbf{3 f}, \mathbf{3 g}$ and $\mathbf{3 j}$ respectively. The NHproton of pyridine ring in $\mathbf{3 h}$ and $\mathbf{3 i}$ are assigned by singlet at 3.37 and 4.47 ppm respectively. Hence, the compounds synthesized were in conformity with the structures postulated.

\section{Antidiabetic Evaluation}

The $\mathrm{LD}_{50}$ values of the synthesized compounds were estimated to be in the range of $100-1000 \mathrm{mg} / \mathrm{kg} \mathrm{b.w.} \mathrm{STZ}$
Table 2. Effect of synthesized test compounds $\mathbf{3}(\mathbf{a}-\mathbf{j})$ on diabetic rats

\begin{tabular}{cccc}
\hline Treatment & \multicolumn{3}{c}{ Blood glucose level (mg/dl) } \\
\cline { 2 - 5 } (100 mg/kg b.w p.o.) & 0 day & $5^{\text {th }}$ day & $10^{\text {th }}$ day \\
\hline Diabetic Control & $274.2 \pm 1.6$ & $272.2 \pm 1.8^{* *}$ & $270.5 \pm 1.7^{* *}$ \\
Glibenclamide $20 \mathrm{mg} / \mathrm{kg}$ & $272.8 \pm 1.6$ & $213.6 \pm 1.35^{* *}$ & $114.0 \pm 1.6^{* *}$ \\
3a & $274.7 \pm 1.9$ & $235.5 \pm 1.65^{* *}$ & $139.5 \pm 1.6^{* *}$ \\
$\mathbf{3 b}$ & $273.2 \pm 1.9$ & $232.7 \pm 2.23^{*}$ & $140.3 \pm 1.4^{*}$ \\
$\mathbf{3 c}$ & $262.5 \pm 4.01$ & $228.0 \pm 2.03^{* *}$ & $132.0 \pm 2.7^{* *}$ \\
$\mathbf{3 d}$ & $268.5 \pm 1.70$ & $231.3 \pm 1.72^{* *}$ & $130.8 \pm 2.42^{* *}$ \\
$\mathbf{3 e}$ & $266.3 \pm 2.9$ & $228.3 \pm 2.38^{* *}$ & $136.0 \pm 2.3^{* *}$ \\
$\mathbf{3 f}$ & $267.5 \pm 3.2$ & $230.7 \pm 2.42^{*}$ & $144.2 \pm 1.9^{*}$ \\
$\mathbf{3 g}$ & $270.7 \pm 2.8$ & $231.5 \pm 2.47^{* *}$ & $148.2 \pm 2.3^{* *}$ \\
$\mathbf{3 h}$ & $271.7 \pm 2.6$ & $232.2 \pm 3.0^{* *}$ & $143.2 \pm 1.6^{* *}$ \\
$\mathbf{3 i}$ & $271.7 \pm 2.5$ & $233.8 \pm 2.2^{* *}$ & $135.7 \pm 2.5^{* *}$ \\
$\mathbf{3 j}$ & $262.5 \pm 4.02$ & $227.7 \pm 2.7^{* *}$ & $132.5 \pm 1.18^{* *}$ \\
\hline Af & & &
\end{tabular}

All the values were expressed as $\mathrm{m} \pm$ S.E.M $(\mathrm{n}=6) .{ }^{*} p<0.05$ and ${ }^{* *} p<0.01$

causes diabetes by the rapid depletion of $\beta$-cells and thereby bring about a reduction of insulin release ${ }^{4}$. The results summarized in Table 2 revealed that all the synthesized compounds exhibited anti diabetic response at the end of ten-day experimental period. From Table 2, it has been found that oral administration of synthesized compounds $\mathbf{3 c}, \mathbf{3 d}, \mathbf{3 e}$ and $\mathbf{3 j}$ caused a more significant reduction in blood glucose than other compounds in diabetic rats. However, the compound 3d at $100 \mathrm{mg} / \mathrm{kg}$ b.w. exerted maximum glucose lowering effects whereas $\mathbf{3 g}$ showed minimum glucose lowering effects. The maximum glucose lowering effects of compound $\mathbf{3 d}$ may be due to the presence of heterocyclic amine (morpholine).

The fundamental mechanism underlying hyperglycemia in diabetes mellitus involves over-production and decreased utilization of glucose by the tissues. The plausible mechanism by which benzothiazole derivatives brought about its hypoglycemic action in diabetic rats might be by potentiating the effect of insulin in plasma by increasing either the pancreatic secretion of insulin from existing beta cells or by its release from bound form. In STZ induced diabetes, induction of diabetes with STZ is associated with characteristic loss of body weight, which is due to increased muscle wasting in diabetes. ${ }^{5}$ The present study has indicated the fact that benzothiazole derivatives have anti diabetic property and further exploitation of benzothiazole core may afford a safe anti diabetic drug.

\section{Pharmacological Evaluation Acute toxicity studies:}

Groups of six albino mice, weighing 20-25 g were fasted 
overnight and treated per orally with test compounds. The dosage was varied from 100 to $1000 \mathrm{mg} \mathrm{kg}^{-1}$ body weight. The animals were observed for $24 \mathrm{~h}$ for any signs of acute toxicity such as increased or decreased motor activity, tremors, convulsion, sedation, lacrimation etc. No such signs, symptoms and mortality were observed even after $24 \mathrm{~h}$. Hence the $\mathrm{LD}_{50}$ cut off value of the test compounds was fixed at $100 \mathrm{mg} / \mathrm{kg}$. b.w and the same dose was considered for evaluation of anti diabetic activity. All the animal experiments were conducted by the approval of Institutional Animal Ethics Committee, Himalayan Pharmacy Institute, East Sikkim, India. During the study period, guidelines of Committee for the Purpose of Control and Supervision of Experiments on Animals (CPCSEA), Institutional Animal Ethics Committee (IAEC) were followed for the maintenance of animals.

\section{Antidiabetic Activity \\ Induction of experimental diabetes by Streptozoto- cin (STZ):}

The rats were injected intraperitoneally with streptozotocin dissolved in sterile normal saline at a dose of $60 \mathrm{mg}$ $\mathrm{kg}^{-1}$ b.w. The animals showing blood glucose range of 200-300 mg dL ${ }^{-1}$ were used for the experiment and the hyperglycemia was confirmed after 72 hours of streptozotocin injection.

\section{Experimental Design}

Animals were divided into 12 groups of 6 animals in each $(n=6)$. Group 1 diabetic animals received $0.5 \%$ carboxy methyl cellulose (CMC) $(1 \mathrm{ml})$; Group 2 diabetic animals received glibenclamide $20 \mathrm{mg} / \mathrm{kg}$. Groups (3-12) diabetic animals received compounds $\mathbf{3 a - 3 j}$ in a single dose of $100 \mathrm{mg} / \mathrm{kg}$ b.w p.o respectively for 7 days continuously.

\section{Blood Glucose Measurement}

Blood was withdrawn from the tail vein each time. At the end of $0,3^{\text {rd }}, 7^{\text {th }}$ and $10^{\text {th }}$ day, blood sample was withdrawn from a tail vein by snipping the tip of the tail and the blood glucose level was measured by Accu Sure Blood Glucose Monitoring System (Dr. Gene Health \& Wellness).

\section{Statistical Analysis}

Values are expressed as mean \pm SEM. Data were analyzed using analysis of variance and group means were compared with Tukey-Kramer Post ANOVA test. The values were considered to be significant at $p<0.05$ and $p<0.01$ level.

\section{EXPERIMENTAL SECTION}

All the chemicals were of synthetic grade and commercially procured from Qualigen, Mumbai, India. Melting points were determined in open capillary method and are uncorrected. IR spectra were recorded on FT-IR8400S, Fourier Transform (Shimadzu) Infrared Spectrophotometer using $\mathrm{KBr}$ disc method. The ${ }^{1} \mathrm{H}-\mathrm{NMR}$ were recorded on BRUKER ADVANCE-II 400 NMR spectrophotometer in DMSO- $d_{6}$ as a solvent and TMS as an internal standard. Mass spectra were recorded on a PEP-SCIUX-APIQ pulsar (electron pre-ionisation) mass spectrometer. Elemental analyses were performed on Perkin-Elmer EAL240 elemental analyzer.

\section{General procedure for the synthesis of 6-chloro-1, 3- benzothiazol-2-amine 1}

To a required amount of chilled glacial acetic acid, KSCN and 4-chloroaniline were added and placed in freezing mixture. The solution was stirred mechanically with dropwise addition of $\mathrm{Br}_{2}$ in glacial acetic acid at such a rate that temperature does not rise above $5^{\circ} \mathrm{C}$. The stirring was continued for an additional $3 \mathrm{hr}$ at $0-10{ }^{\circ} \mathrm{C}$ and the separated hydrochloride salt was filtered, washed with acetic acid and dried. It was dissolved in hot water and neutralized with aqueous ammonia solution (25\%). The resulting precipitate was filtered, washed with water and recrystallized from methanol to obtain pure 6-chloro-1, 3benzothiazol-2-amine.

\section{Synthesis of 2-chloro-N-(6-chlorobenzothiazol-2-yl) acetamide 2}

Equimolar quantity of compound $\mathbf{1}$ and chloroacetyl chloride in sufficient quantity of chloroform was refluxed in the presence of $\mathrm{K}_{2} \mathrm{CO}_{3}$ for about 10 hours. Excess solvent was removed in vacuum and the residue thus obtained was washed with $5 \% \mathrm{NaHCO}_{3}$ and subsequently with water. The resulting crude product was dried and recrystallized from ethanol to furnish white crystal.

\section{Synthesis of N-(6-chlorobenzothiazol-2-yl)-2-(substi- tuted amino) acetamide $3(\mathbf{a}-\mathbf{j})$}

To a solution of compound $2(0.01 \mathrm{~mol})$ in $25 \mathrm{ml}$ of absolute alcohol was added different secondary and primary amine $(0.01 \mathrm{~mol})$. The mixture was refluxed on water bath for 4-6 hours and the completion of reaction was checked by TLC. The crude product thus obtained was filtered, dried and recrystallized from aqueous alcohol. 
$N$-(6-chlorobenzo[d]thiazol-2-yl)-2-(dimethylamino) acetamide (3a):

Colorless crystal; UV (nm) 225; IR $\left(\mathrm{KBr}, \mathrm{cm}^{-1}\right) 3460$ $(\mathrm{N}-\mathrm{H}), 3063\left(\mathrm{C}-\mathrm{H}\right.$ str of $\left.\mathrm{CH}_{2}\right), 1531(\mathrm{C}=\mathrm{O}), 1444(\mathrm{C}=\mathrm{N})$; ${ }^{1} \mathrm{HNMR}\left(400 \mathrm{~Hz}\right.$, DMSO- $\left.d_{6}\right): \delta 7.61(\mathrm{~s}, 1 \mathrm{H}, \mathrm{NH}), \delta 2.50$ $\left(\mathrm{s}, 2 \mathrm{H}, \mathrm{CH}_{2}\right), \delta 3.37\left(\mathrm{~s}, 6 \mathrm{H},\left(\mathrm{CH}_{3}\right)_{2}\right), \delta$ 7.19-7.76 (m, $3 \mathrm{H}$, Ar-H); Mass (m/z) 269; Ana. Calcd. $\mathrm{C}_{11} \mathrm{H}_{12} \mathrm{ClN}_{3} \mathrm{OS} \mathrm{C}$, 48.98; H, 4.48; N, 15.58, found C, 49.38; H, 4.58; N, 15 . $48 \%$.

\section{$\mathrm{N}$-(6-chlorobenzo[d]thiazol-2-yl)-2-(diethylamino)} acetamide (3b):

Colorless crystal; UV (nm) 225; IR $\left(\mathrm{KBr}, \mathrm{cm}^{-1}\right) 3456$ $(\mathrm{N}-\mathrm{H}), 3091\left(\mathrm{CH}\right.$ str of $\left.\mathrm{CH}_{2}\right), 1637(\mathrm{C}=\mathrm{O}), 1533(\mathrm{C}=\mathrm{N})$; ${ }^{1} \mathrm{H}$ NMR $\left(400 \mathrm{~Hz}, \mathrm{DMSO}-d_{6}\right): \delta 7.61(\mathrm{~s}, \mathrm{IH}, \mathrm{NH}), \delta 2.50$ (s, $\left.4 \mathrm{H},\left(\mathrm{CH}_{2}\right)_{2}\right), \delta 3.38\left(\mathrm{~s}, 6 \mathrm{H},\left(\mathrm{CH}_{3}\right)_{2}\right) 7.19-7.76(\mathrm{~m}, 3 \mathrm{H}$, Ar-H); Mass (m/z) 298; Ana. Calcd. $\mathrm{C}_{13} \mathrm{H}_{16} \mathrm{ClN}_{3} \mathrm{OS} \mathrm{C}$, $52.43 ; \mathrm{H}, 5.42 ; \mathrm{N}, 14.11$, found $\mathrm{C}, 52.83 ; \mathrm{H}, 5.12 ; \mathrm{N}$, $14.51 \%$.

N-(6-chlorobenzo[d]thiazol-2-yl)-2-(bis (2-hydroxyethyl) amino) acetamide $(3 \mathrm{c})$ :

Colorless crystal; UV (nm) 226; IR (KBr, $\left.\mathrm{cm}^{-1}\right) 3458$ (O-H), 3271(N-H), $3093\left(\mathrm{C}-\mathrm{H}\right.$ str of $\left.\mathrm{CH}_{2}\right), 1637(\mathrm{C}=\mathrm{O})$, 1533(C=N); ${ }^{1} \mathrm{H}$ NMR (400 Hz, DMSO- $\left.d_{6}\right): \delta 7.60(\mathrm{~s}, 1 \mathrm{H}$, $\mathrm{NH}), \delta 3.39\left(\mathrm{~s}, 2 \mathrm{H}, \mathrm{CH}_{2}\right), \delta 2.49\left(\mathrm{~s}, 8 \mathrm{H}, 2 \times\left(\mathrm{CH}_{2}\right)_{2}\right), \delta 2.48$ (s, $2 \mathrm{H}, 2 \times \mathrm{OH}), \delta$ 7.17-7.74 (m, 3H, Ar-H); Mass (m/z) 329; Ana. Calcd. $\mathrm{C}_{13} \mathrm{H}_{16} \mathrm{ClN}_{3} \mathrm{O}_{3} \mathrm{~S} \mathrm{C}$, 47.34; H, 4.89; N, 12.74; found $\mathrm{C}, 47.74 ; \mathrm{H}, 4.99 ; \mathrm{N}, 12.64 \%$.

$\mathrm{N}$-(6-chlorobenzo[d]thiazol-2-yl)-2-morpholinoacetamide (3d):

Colorless crystal; UV (nm) 215; IR $\left(\mathrm{KBr}, \mathrm{cm}^{-1}\right) 3329$ $(\mathrm{N}-\mathrm{H}), 3080\left(\mathrm{C}-\mathrm{H}\right.$ str of $\left.\mathrm{CH}_{2}\right), 1695(\mathrm{C}=\mathrm{O}), 1599(\mathrm{C}=\mathrm{N})$; ${ }^{1} \mathrm{H}$ NMR $\left(400 \mathrm{~Hz}, \mathrm{DMSO}-d_{6}\right): \delta$ 7.14(s, $\left.1 \mathrm{H}, \mathrm{NH}\right), \delta 3.62$ (s, $\left.2 \mathrm{H}, \mathrm{CH}_{2}\right), \delta 2.52\left(\mathrm{~s}, 4 \mathrm{H}, 2 \times \mathrm{CH}_{2}\right.$ of morpholine), $\delta$ $3.55\left(\mathrm{~s}, 4 \mathrm{H}, 2 \times \mathrm{CH}_{2}\right.$ of morpholine), $\delta 7.42-8.12(\mathrm{~m}, 3 \mathrm{H}$, Ar-H); Mass (m/z) 312; Ana. Calcd. $\mathrm{C}_{13} \mathrm{H}_{14} \mathrm{ClN}_{3} \mathrm{O}_{2} \mathrm{~S} \mathrm{C}$, $50.08 ; \mathrm{H}, 4.53 ; \mathrm{N}, 13.48$; found $\mathrm{C}, 50.48 ; \mathrm{H}, 4.83 ; \mathrm{N}$, $13.28 \%$.

$N$-(6-chlorobenzo[d]thiazol-2-yl)-2-(piperidin-1-yl) acetamide (3e):

Pale yellow powder; $\mathrm{UV}(\mathrm{nm}) 224 ; \mathrm{IR}\left(\mathrm{KBr}, \mathrm{cm}^{-1}\right) 3460$ $(\mathrm{N}-\mathrm{H}), 3265\left(\mathrm{C}-\mathrm{H}\right.$ str of $\left.\mathrm{CH}_{2}\right), 1639(\mathrm{C}=\mathrm{O}), 1433(\mathrm{C}=\mathrm{N})$, ${ }^{1} \mathrm{H}$ NMR $\left(400 \mathrm{~Hz}, \mathrm{DMSO}-d_{6}\right): \delta 7.60(\mathrm{~s}, 1 \mathrm{H}, \mathrm{NH}), \delta 3.35$ (s, $2 \mathrm{H}, \mathrm{CH}_{2}$ ), $\delta 1.53$ (s, $8 \mathrm{H}, 4 \times \mathrm{CH}_{2}$ of piperidine), $\delta 2.50$ (s, $2 \mathrm{H}, \mathrm{CH}_{2}$ of piperidine), $\delta$ 7.19-7.77 (m, $\left.3 \mathrm{H}, \mathrm{Ar}-\mathrm{H}\right)$; Mass (m/z) 309; Ana. Calcd. $\mathrm{C}_{14} \mathrm{H}_{16} \mathrm{ClN}_{3} \mathrm{OS} \mathrm{C}, 54.27 ; \mathrm{H}$, $5.21 ; \mathrm{N}, 13.56$; found $\mathrm{C}, 54.67 ; \mathrm{H}, 4.81 ; \mathrm{N}, 13.76 \%$.

$\mathrm{N}$-(6-chlorobenzo[d]thiazol-2-yl)-2-(4-fluorophenylamino) acetamide (3f):

Colorless powder; UV (nm) 218; IR $\left(\mathrm{KBr}, \mathrm{cm}^{-1}\right) 3365$
$(\mathrm{N}-\mathrm{H}), 3178\left(\mathrm{C}-\mathrm{H}\right.$ str of $\left.\mathrm{CH}_{2}\right), 1600(\mathrm{C}=\mathrm{O}), 1521(\mathrm{C}=\mathrm{N})$; ${ }^{1} \mathrm{H}$ NMR $\left(400 \mathrm{~Hz}, \mathrm{DMSO}-d_{6}\right): \delta 8.13(\mathrm{~s}, 1 \mathrm{H}, \mathrm{NH}), \delta 3.36$ (s, $\left.2 \mathrm{H}, \mathrm{CH}_{2}\right), 4.06$ (s, $\left.1 \mathrm{H}, \mathrm{Ar}-\mathrm{NH}\right), \delta 6.55-8.13(\mathrm{~m}, 3 \mathrm{H}$, Ar-H), $\delta$ 7.73-7.43 (m, 4H, Ar-H); Mass (m/z) 336; Ana. Calcd. $\mathrm{C}_{15} \mathrm{H}_{11} \mathrm{ClFN}_{3} \mathrm{OS} \mathrm{C}$, 53.65; H, 3.30; N, 12.51; found C, 53.25; H, 3.6; N, 12.91\%.

$\mathrm{N}$-(6-chlorobenzo[d]thiazol-2-yl-2-(3-chlorophenylamino) acetamide (3g):

Colorless powder; UV (nm) 282; IR ( $\left.\mathrm{KBr}, \mathrm{cm}^{-1}\right), 3379$ $(\mathrm{N}-\mathrm{H}), 3176\left(\mathrm{C}-\mathrm{H}\right.$ str of $\left.\mathrm{CH}_{2}\right), 1668(\mathrm{C}=\mathrm{O}), 1541(\mathrm{C}=\mathrm{N})$; ${ }^{1} \mathrm{H}$ NMR (400 Hz, DMSO- $\left.d_{6}\right): \delta 7.73$ (s, 1H, NH), $\delta 4.04$ (s, $\left.2 \mathrm{H}, \mathrm{CH}_{2}\right), \delta 4.46(\mathrm{~s}, \mathrm{IH}, \mathrm{Ar}-\mathrm{NH}), \delta 6.52-8.14(\mathrm{~m}, 7 \mathrm{H}$, Ar-H); Mass (m/z) 352; Ana. Cald. $\mathrm{C}_{15} \mathrm{H}_{11} \mathrm{Cl}_{2} \mathrm{~N}_{3} \mathrm{OS} \mathrm{C}$, $51.15 ; \mathrm{H}, 3.15 ; \mathrm{N}, 11.93$; found $\mathrm{C}, 51.05 ; \mathrm{H}, 3.45 ; \mathrm{N}$, $11.73 \%$.

$\mathrm{N}$-(6-chlorobenzo[d]thiazol-2-yl)-2-(pyridin-4-ylamino) acetamide (3h):

Colorless crystal; UV (nm) 224; IR (KBr, cm $\left.{ }^{-1}\right), 3456$ $(\mathrm{N}-\mathrm{H}), 3269\left(\mathrm{C}-\mathrm{H}\right.$ str of $\left.\mathrm{CH}_{2}\right), 1637(\mathrm{C}=\mathrm{O}), 1533(\mathrm{C}=\mathrm{N})$; ${ }^{1} \mathrm{H}$ NMR (400 Hz, DMSO- $\left.d_{6}\right): \delta 7.61$ (s, $\left.1 \mathrm{H}, \mathrm{NH}\right), \delta 2.51$ (s, $\left.2 \mathrm{H}, \mathrm{CH}_{2}\right), \delta 3.37(\mathrm{~s}, 1 \mathrm{H}$, pyridine-NH), $\delta$ 7.19-7.22 (m, $3 \mathrm{H}, \mathrm{Ar}-\mathrm{H}), \delta$ 7.28-7.77 (m, 4H, Het. Ar-H); Mass (m/z) 318; Ana. Cald. $\mathrm{C}_{14} \mathrm{H}_{11} \mathrm{ClN}_{4} \mathrm{OS} \mathrm{C}, 52.75 ; \mathrm{H}, 3.48 ; \mathrm{N}$, 17.58; found $\mathrm{C}, 51.43 ; \mathrm{H}, 3.52 ; \mathrm{N}, 17.43 \%$.

$\mathrm{N}$-(6-chlorobenzo[d]thiazol-2-yl)-2-(pyridin-2-ylamino) acetamide (3i):

Brick red powder; UV (nm) 224; IR $\left(\mathrm{KBr}, \mathrm{cm}^{-1}\right), 3454$ $(\mathrm{N}-\mathrm{H}), 3416\left(\mathrm{C}-\mathrm{H}\right.$ str of $\left.\mathrm{CH}_{2}\right), 1699(\mathrm{C}=\mathrm{O}), 1535(\mathrm{C}=\mathrm{N})$; ${ }^{1} \mathrm{H}$ NMR (400 Hz, DMSO- $\left.d_{6}\right): \delta 7.60$ (s, $\left.1 \mathrm{H}, \mathrm{NH}\right), \delta 3.36$ $\left(\mathrm{s}, 2 \mathrm{H}, \mathrm{CH}_{2}\right), \delta 4.47(\mathrm{~s}, 1 \mathrm{H}$, pyridine- $\mathrm{NH}), \delta$ 7.19-7.31 (m, $3 \mathrm{H}, \mathrm{Ar}-\mathrm{H}), \delta$ 7.46-8.15 (m, 4H, Het. Ar-H); Mass (m/z) 318; Ana. Cald $\mathrm{C}_{14} \mathrm{H}_{11} \mathrm{ClN}_{4} \mathrm{OS} \mathrm{C}$, 52.75; H, 3.48; N, 17.58; found $\mathrm{C}, 52.43 ; \mathrm{H}, 3.48 ; \mathrm{N}, 17.43 \%$.

$\mathrm{N}$-(6-chlorobenzo[d]thiazol-2-yl)-2-(4-sulfanilido) acetamide $(3 \mathrm{j})$ :

Colorless crystal; UV (nm) 281; IR $\left(\mathrm{KBr}, \mathrm{cm}^{-1}\right), 3180$ $(\mathrm{N}-\mathrm{H}), 2993\left(\mathrm{C}-\mathrm{H}\right.$ str of $\left.\mathrm{CH}_{2}\right), 1668(\mathrm{C}=\mathrm{O}), 1541(\mathrm{C}=\mathrm{N})$; ${ }^{1} \mathrm{H}$ NMR $\left(400 \mathrm{~Hz}, \mathrm{DMSO}-d_{6}\right): \delta 8.15(\mathrm{~s}, 1 \mathrm{H}, \mathrm{NH}), \delta 3.38$ (s, $\left.2 \mathrm{H}, \mathrm{CH}_{2}\right), \delta 4.49(\mathrm{~s}, 1 \mathrm{H}, \mathrm{NH}), \delta 7.46\left(\mathrm{~m}, 2 \mathrm{H}, \mathrm{SO}_{2} \mathrm{NH}_{2}\right)$, 87.75-8.15 (m, 7H, Ar-H); Mass (m/z) 397; Ana. Cald $\mathrm{C}_{15} \mathrm{H}_{13} \mathrm{ClN}_{4} \mathrm{O}_{3} \mathrm{~S}_{2} \mathrm{C}, 45.40 ; \mathrm{H}, 3.30 ; \mathrm{N}, 14.12$; found $\mathrm{C}$, 45.43 ; H, 3.44; N, $14.22 \%$.

Acknowledgements. The authors are thankful to the Dr. H.P.Chhetri, Director, Himalayan Pharmacy Institute, Majhitar, East Sikkim, India who provided the facilities to carry out the research work. The authors are also grateful to Director, IICB for providing spectral data. 


\section{REFERENCES}

1. Geewananda, G. P.; Shigeo, K.; Sarath, P. G.; Oliver, J. M.; Frank, E. K. J. Am. Chem. Soc. 1988, 110(14), 4856.

2. Geewananda, G. P.; Shigeo, K.; Neal, S.B. Tetrahedron Lett. 1989, 30, 4359.

3. Gunawardana, G. P.; Koehn, F. E.; Lee, A. Y.; Clardy, J.; He, H. Y.; Faulkenr, J. D. J. Org. Chem. 1992, 57(5), 523.

4. Carroll, A. R.; Scheuer, P. J. J. Org. Chem. 1990, 55(14), 4426.

5. Bryson, M.; Fulton, B.; Benfield, P. Drugs. 1996, 52, 549.

6. Chopade, R. S.; Bahekar, R. H.; Khedekar, P. B.; Bhusari, K. P.; Rao, A.R.R. Arch. Pharm. Pharm. Med. Chem. 2002, 8, 381.

7. Yogeeswari, P.; Srisam, D.; Suniljit, L.; Kumar, S.; Stables, J. Eur. J. Med. Chem. 2002, 37, 231.

8. Yogeeswari, P.; Sriram, D.; Mehta, S.; Nigam, D.; Kumar, M.; Murugesan, S. J. Stables II, Farmaco. 2005, 60, 1.

9. Siddiqui, N.; Pandeya, S.; Khan, S.; Stables, J.; Rana, A.; Alam, M.; Arshad, M.; Bhat, M. Bioorg. Med. Chem. Lett. 2007, 17, 255.

10. Siddiqui, N.; Rana, A.; Khan, S.; Bhat, M.; Haque, S. Bioorg. Med. Chem. Lett. 2007, 17, 4178.

11. Hays, S. J.; Rice, M. J.; Ortwine, D. F.; Johnson, G.; Schwartz, R. D.; Boyd, D. K.; opeland, L. F.; Vartanian, M. G.; Boxer, P. A. J. Pharm. Sci. 1994, 83, 1425.

12. He, Y.; Benz, A.; Fu, T.; Wang, M.; Covey, D. F.; Zorumski, C. F.; Mennick, S. Neuropharmacology 2002, 42, 199.

13. Gurupadayya, B. M.; Gopal, M.; Padmashali, B.; Vaidya, V. P. Int. J. Heterocyclic Chem. 2005, 15, 169.

14. Sawhney, S. N.; Arora, S. K.; Singh, J. V.; Bansal, O. P.; Singh, S. P. Indian J. Chem. 1978, 16B, 605.

15. Foscolos, G.; Tsatsas, G.; Champagnac, A.; Pommier, M. Ann. Pharm. Fr. 1977, 35, 295.

16. (a) Siddiqui, N.; Alam, M.; Siddiqui, A. A. Asian. J. Chem. 2004, 16, 1005. (b) Bensimon, G.; Lacomblez, L.; Meininger, V. New Engl. J. Med. 1994, 330, 585.

17. Bensimon, G.; Lacomblez, L.; Meininger, V. New Engl. J. Med. 1994, 330, 585.

18. Paget, C. J.; Kisner, K.; Stone, R. L.; Delong, D. C. J. Med. Chem. 1969, 12, 1016.

19. Vicini, P.; Gernonikaki, A.; Incerti, M.; Busonera, B.; Poni, G.; Cabras, C. A.; Colla, P. L. Bioorg. Med. Chem. 2003, 11, 4785.

20. Caleta, I.; Kralj, M.; Branimir Bertosa, B.; Sanja Tomic, S.; Pavlovic, G.; Pavelic, K.; Karminski-Zamola, G. J. Med. Chem. 2009, 52, 1744.

21. Chung, Y.; Shin, Y. K.; Zhan, C. G.; Lee, S.; Cho, H. Arch. Pharmacol. Res. 2004, 27, 893.

22. Yoshida, M.; Hayakawa, I.; Hayashi, N.; Agatsuma, T.; Oda, Y.; Tanzawa, F.; Iwasaki, S.; Koyama, K.; Furukawa, H.; Kurakata, S. Bioorg. Med. Chem. Lett. 2005, 15, 3328.

23. Bradshaw, T. D.; Stevens, M. F. G.; Westwell, A. D. Curr. Med. Chem. 2001, 8, 203.

24. Chua, M. S.; Shi, D. F.; Wrigley, S.; Bradshaw, T. D.; Hutch- inson, I.; Shaw, P. N.; Barrett, D. A.; Stanley, L. A.; Stevens, M. F. G. J. Med. Chem. 1999, 42, 381.

25. O'Brien, S. E.; Browne, H. L.; Bradshaw, T. D.; Westwell, A. D.; Stevens, M. F. G.; Laughton, C. A. Org. Biomol. Chem. 2003, 1, 493.

26. Bradshaw, T. D.; Wrigley, S.; Shi, D. F.; Schulz, R. J.; Paull, K. D.; Stevens, M. F. G. Br. J. Cancer 1998, 77, 745.

27. Kashiyama, E.; Hutchinson, I.; Chua, M. S.; Stinson, S. F.; Phillips, L. R.; Kaur, G.; Sausville, E. A.; Bradshaw, T. D.; Westwell, A. D.; Stevens, M. F. G. J. Med. Chem. 1999, 42, 4172.

28. Hutchinson, I.; Chua, M. S.; Browne, H. L.; Trapani, V.; Bradshaw, T. D.; Westwell, A. D.; Stevens, M. F. G. J. Med. Chem. 2001, 44, 1446.

29. Shi, D. F.; Bradshaw, T. D.; Wrigley, S.; McCall, C. J.; Lelieveld, P.; Stevens, M. F. G. J. Med. Chem. 1996, 39, 3375.

30. Lion, C. J.; Matthews, C. S.; Wells, G.; Bradshaw, T. D.; Stevens, M. F. G.; Westwell, A. D. Bioorg. Med. Chem. Lett. 2006, 16, 5005.

31. Mortimer, C. S.; Wells, G.; Crochard, P. J.; Stone, E. L.; Bradshaw, T. D.; Stevens, A. D.; Westwell, M. F. G. $J$. Med. Chem. 2006, 49, 179.

32. Wells, G.; Berry, J. M.; Bradshaw, T. D.; Burger, A. M.; Seaton, A.; Wang, B.; Westwell, A. D.; Stevens, M. F. G. J. Med. Chem. 2003, 46, 532.

33. Hutchinson, I.; Jennings, S. A.; Vishnuvajjala, B. R.; Westwell, A. D.; Stevens, M. F. G. J. Med. Chem. 2002, 45, 744 .

34. Khadse, B. G.; Sengupta, S. R. Indian J. Chem. 1993, Sec-B, 407.

35. Palmer, F. J.; Trigg, R. B.; Warrington, J. V. J. Med. Chem. 1971, 14, 248.

36. Gurupadaiah, B. M.; Jayachandran, E.; ShivaKumar, B.; Nagappa, A. N.; Nargund, L. V. G. Indian J. Heterocycl. Chem. 1998, 7, 213.

37. Gopkumar, P.; Shivakumar, B.; Jayachandran, E.; Nagappa, A. N.; Nargund, L. V. G.; Gurupadaiah, B. M. Indian J. Heterocycl. Chem. 2001, 11, 39.

38. Burger, A.; Sawhey, S. N. J. Med. Chem. 1968, 11, 270.

39. Jayachandran, E.; Bhatia, K.; Naragud, L. V. G.; Roy, A. Indian Drugs 2003, 40, 408.

40. Weekes, A. A.; Westwell, A. D. Curr. Med. Chem. 2009, $16,2430$.

41. Henriksen, G.; Hauser, A. I.; Westwell, A. D.; Yousefi, B. H.; Schwaiger, M.; Drzega, A.; Wester, H. J. J. Med. Chem. 2007, 50, 1087.

42.Mathis, C. A.; Wang, Y.; Holt, D. P.; Haung, G. F.; Debnath, M. L.; Klunk, W. E. J. Med. Chem. 2003, 46, 2740.

43. Wang, X.; Sarris, K.; Zhang, K. Kage, D.; Brown, S. P.; Kolasa, T.; Surowy, C.; ElKouhen, O. F.; Muchmore, S. W.; Brioni, J. D.; Stewart, A. O. J. Med. Chem. 2009, 52, 170.

44. Suter, H.; Zutter, H. Helv. Chim. Acta 1967, 50, 1084.

45. Diaz, H. M.; Molina, R. V.; Andrade, R. O.; Coutino, D. 
D.; Franco, L. M.; Webster, S. P.; Binnie, M.; Soto, S. E.; Barajas, M. I.; Rivera, I. L.; Vazquez, G. N. Bioorg. Med. Chem. Lett. 2008, 18, 2871.

46. Nitta, A.; Fujii, H.; Sakami, S.; Nishimura, Y.; Ohyama, T.; Satoh, M.; Nakaki, J.; Satoh, S.; Inada, C.; Kozono, H.; Kumagai, H.; Shimamura, M.; Fukazawa, T.; Kawai, H. Bioorg. Med. Chem. Lett. 2008, 15, 5435.

47. Vazquez, G. N.; Paoli, P.; Rivera, I. L.; Molina, R. V.; Franco, J. L. M.; Andrade, R. O.; Soto, S. E.; Camici, G.; Coutiño, D. D.; Ortiz, I. G.; Mayorga, K. M.; Díaz, H. M. Bioorg. Med. Chem. Lett. 2009, 17, 3332.

48. Su, X.; Vicker, N.; Ganeshapillai, D.; Smith, A.; Purohit, A.; Reed, M. J.; Potter, B. V. Mol. Cell Endocrinol. 2006,
248, 214.

49. Barf, T.; Vallgarda, J.; Emond, R.; Haggstrom, C.; Kurz, G.; Nygren, A.; Larwood, V.; Mosialou, E.; Axelsson, K.; Olsson, R.; Engblom, L.; Edling, N.; Ronquist-Nii, Y.; Ohman, B.; Alberts, P.; Abrahmsen, L. J. Med. Chem. 2002, 45, 3813.

50. Fujieda, H.; Usui, S.; Suzuki, T.; Nakagawa, H.; Ogura, M.; Makishima, M.; Miyata, N. Bioorg. Med. Chem. Lett. 2007, 17, 4351.

51. Jeon, R.; Kim, Y. J.; Cheon, Y.; Ryu, J. H. Arch. Pharmacal Res. 2006, 29, 394.

52. Pattan, S. R.; Suresh, C.; Pujar, V. D.; Reddy, V. V. K.; Rasal, V. P.; Koti, B. C. Indian. J. Chem. 2005, 44B, 2404. 